\title{
BaccuS: A framework for sustainability in the wine industry I BaccuS : un framework pour le développement durable dans la vitiviniculture
}

\author{
Shana Sabbado Flores ${ }^{1, a}$ et Rosa Maria Vieira Medeiros ${ }^{2}$ \\ ${ }^{1}$ Federal Institute of Education, Science and Technology of Rio Grande do Sul (IFRS) - campus Restinga, Rua Alberto Hoffmann 285, \\ CEP 91791-508, Porto Alegre, RS, Brazil \\ ${ }^{2}$ Federal University of Rio Grande do Sul - Postgraduate Program of Geography (PosGea), Av. Bento Gonçalves, 9500/43113/203, \\ CEP 91540-000, Porto Alegre, RS, Brazil
}

\begin{abstract}
The BaccuS is a proposal of framework intended to support the internalization of sustainability principles in the wine industry, enhancing the actors' performance in this sense. The framework was built from two exploratory studies in Brazilian wine regions of Campanha Gaúcha (RS) and Vale do São Francisco (BA/PE) and preliminary studies which involved technical visits in three countries (France, Italy and Spain) and frameworks' observations in another five (South Africa, Australia, New Zealand, USA and Chile). The BaccuS is structured on a matrix basis, with an axis corresponding to dimensions and other to guidelines, both in ascending order of complexity and development. The five sustainability dimensions (environment, economic, social, political- institutional and territorial) and the four guidelines (management, articulation and cooperation, innovation and learning and sustainability) are articulated by eighteen topics that represent areas to act to promote the sustainability in the wine territories. Each topic was unfolded in indicators. Additionally, ten synthesis indicators specify initiatives or programs that can be implemented to impact positively on several indicators. The BaccuS framework can be applied in several scales, in the winery or the territory.
\end{abstract}

\section{Du développement durable à la viticulture durable}

Le développement durable est un approche complexe qui nécessite de repenser nos activités, outils et bases méthodologiques. Même si l'urgence de cette question est un consensus relatif, vue la dégradation de l'environnement et ses implications sociales, il peut être observée une manque en termes de propositions concrètes.

La répercussion internationale dans le contexte contemporain date des années ' 60 , soulignant le travail de Rachel Carson et son livre « Silent Spring », qui discute sur l'intensification de l'utilisation des technologies chimiques et mécaniques et ses effets sur la santé humaine et sur la biodiversité [1]. Plus tard, le travail de Ignacy Sachs a défini le concept de l'écodéveloppement [2]. Le concept le plus populaire a été introduit dans les années '80 par le rapport intitulé « Our Common Future », appelé aussi le rapport de Brundtland, définissant le développement durable comme « [...] un développement qui répond aux besoins du présent sans compromettre la capacité des générations futures à répondre à leurs propres besoins »[3]. Cette approche a lancé la notion de développement durable fondée sur les aspects environnementaux, sociaux et économiques.

En termes de base théorique, les chercheurs soulignent une approche interdisciplinaire pour soutenir les propositions du développement durable. Par exemple, Leff discute que la dimension de l'environnement, de la même manière

a e-mail : shana.flores@restinga.ifrs.edu.br que les catégories société ou nature, n'est pas un sujet d'un seul domaine d'étude et nécessite l'acceptation et l'articulation du travail interdisciplinaire et à multi-échelle, en respectant les connaissances construites dans des cadres conceptuelles spécifiques [4,5]. Egalement, Morin recommande une vue interdisciplinaire (ou transdisciplinaire) pour soutenir l'éducation environnementale et l'approche du développement durable, afin de comprendre le tout et ses parties, en utilisant la complexité comme directive principale.

Un autre point de convergence est l'échelle: même si les problèmes sont globaux, c'est au niveau local que les effets sont perçus et les mesures peuvent être prises - ce qui mettre en évidence le territoire. La conviction dans l'échelle territoriale a des raisons objectives, économiques et pragmatiques [6], en soulignant l'importance de considérer les spécificités et les contextes locales dans les propositions par rapport au développement durable. Dans un autre point de vue, la crise environnementale n'est pas une crise de nature elle-même, mais une crise dans la relation entre l'homme et la nature, liée à la production de territorialités, donc une crise du territoire [7]. Cette approche est significative dans les activités où la dimension territoriale est importante, ce qui est le cas de l'industrie du vin, en considérant la notion de terroir, par exemple.

La discussion sur le développement durable arrive à de nombreux secteurs de la société, motivés par les pressions de la société et des stakeholders, l'engagement des consommateurs dans les questions environnementales, les normes de qualité, les exigences légales, la vision à

(C) The Authors, published by EDP Sciences. This is an Open Access article distributed under the terms of the Creative Commons Attribution License 4.0 (http://creativecommons.org/licenses/by/4.0/). 
long terme ou même la réduction des coûts et des enjeux liés à la compétitivité. Dans l'industrie du vin, il doit être considéré aussi que les vignobles sont des cultures avec un impact environnemental importante. Pour illustrer cela, en 2002, les vignobles représentaient $15,6 \%$ du marché des pesticides français, avec seulement $3,2 \%$ de la surface agricole du pays [8]. Ainsi, les enjeux du développement durable peuvent intégrer l'industrie du vin de différentes façons.

Regardant les définitions, la FAO (l'Organisation pour 1'Alimentation et l'Agriculture des Nations Unies) a adopté, à partir de 1989, le concept de « l'agriculture durable et de développement rural »sur la base de l'équilibre entre les dimensions environnementale, sociale et économique, soulignant la conservation de l'environnement (sol, eau et ressources génétiques animales et végétales), la viabilité économique et l'acceptation sociale [9]. Pour les vins, l'OIV a défini la viticulture durable comme un « [...] approche globale à l'échelle des systèmes de production et de transformation des raisins, associant à la fois la pérennité économique des structures et des territoires, l'obtention de produits de qualité, la prise en compte des exigences d'une viticulture de précision, des risques liés à l'environnement, à la sécurité des produits et à la santé des consommateurs et la valorisation des aspects patrimoniaux, historiques, culturels, écologiques et paysagers »[10]. Dans cette définition, il peut être noté que les questions environnementales et économiques sont prises en compte, mais également les aspects immatériels, qui mettent en évidence cette caractéristique particulière des vins et ses enjeux liés au terroir et à l'identité.

Au-delà des définitions, de nombreuses propositions ont été faites afin de systématiser les initiatives de développement durable dans différents domaines. Ils prennent en général la forme de cadres, des lignes directrices, des programmes nationaux, locaux ou sectoriels, des labels ou des certifications, des empreintes et des auto-évaluations. Également, l'agriculture a des méthodes spécifiques, telles que la agriculture biologique et biodynamique ou de l'agriculture raisonnée. Pour les régions viticoles, les guides de développement durable et les évaluations sont de plus en plus d'importantes, soit comme un moyen de positionnement de la région viticole en relation avec les enjeux du développement durable, pour le marché de l'exportation, soit comme un outil pour aligner la supply chain et de promouvoir la compétitivité $\mathrm{du}$ produit et les processus d'innovation. Les principaux producteurs se tournent pour adopter des directives et normes liées au développement durable, comme on peut le voir dans le Tableau 1.

Cet article vise à présenter BaccuS [22], un cadre pour internaliser le développement durable dans l'industrie du vin. Le nom BaccuS a été choisi pour rappeler le Dieu romain du vin et de mettre en évidence le $\langle S »$, de sustainability. Le cadre répond à trois enjeux dans l'évaluation de performance vis-à-vis du développement durable: l'échelle, les questions territoriales et le parcours d'apprentissage. En termes d'échelle, BaccuS peut être appliqué dans la cave ainsi que le territoire du vin, fournissant une évaluation individuelle et / ou une perspective collective. En outre, le cadre considère la participation locale avec les associations et les parties prenantes, ainsi que la gouvernance de la région. L'approche territoriale contribue à une perspective transversale et de synthèse.

Le chemin d'apprentissage était considéré un facteur essentiel pour permettre aux caves ou aux producteurs avec une échèle plus petite de production à introduire des cadres liés au développement durable. La plupart des méthodologies ou certifications dans le contexte international sont complexes et ont besoin d'une structure importante, en termes de connaissances, de formation et de gestion de l'information, pour être appliqués, ce qui est une barrière pour sa diffusion, surtout pour celle publique mentionnée au-dessus. En ce sens, BaccuS a été construit dans une base matriciel qui permet démarrer à partir d'un seul thème (comme l'eau, les pratiques agricoles, système de gestion ou de gouvernance, par exemple) et d'identifier les liens avec les autres thèmes et dimensions. En outre, dix indicateurs de synthèse sont proposés et peuvent être utilisés comme des programmes pour introduire le développement durable, car ils agissent comme des « multiplicateurs »dans le sens qui peuvent impacter sur différents thèmes et dimensions.

Le cadre a été construit à partir des deux études exploratoires dans des régions viticoles et des études préliminaires qui impliquaient des visites techniques dans trois pays (France, Italie et Espagne) et les observations des cadres dans cinq autres (Afrique du Sud, Australie, Nouvelle-Zélande, États-Unis et le Chili). Les matériaux et les méthodes utilisées pour développer la recherche sont présentées dans la Sect. 2 et la Sect. 3 est consacrée à présenter le cadre BaccuS.

\section{Matériels et méthodes}

La structure da la recherche a été construite sur une base qualitative de nature exploratoire. La recherche a eu lieu entre 2012 et 2015, avec le support de la CAPES, dans le cadre du programme «Sciences sans Frontières », de la Chaire-UNESCO «Culture et Traditions du Vin » et de l'Institut Fédéral du Rio Grande do Sul (IFRS).

La recherche a compté avec trois phases : (1) des concepts et des contextes de la viticulture durable; (2) la systématisation des données et l'identification des potentiels ; et (3) la proposition du BaccuS. La première phase a compris la recherche théorique sur le thème, ainsi que des études préliminaires sur les cadres pour la viticulture durable et des exemples des pratiques au vignoble et aux caves. Pour cela, des visites techniques ont été faites dans trois pays (France, Italie et Espagne), ainsi que la recherche documentaire et bibliographique dans cinq autres (Afrique du Sud, Australie, Nouvelle-Zélande, États-Unis et le Chili). Le choix des pays a considéré ceux indiques par des experts contactés et les principaux dans les publications internationales [23,24].

Le référentiel empirique principal sont deux régions viticoles brésiliennes considérés émergents : la Campanha Gaúcha et la Vale do São Francisco. Le choix de ces régions (et non la Serra Gaúcha, la principale région viticole du Brésil) est justifié par plusieurs raisons, parmi lesquelles : les deux sont des régions en expansion, favorables pour l'application des techniques nouvelles, avec disponibilité de terrain, ce qui rendrait possible un meilleur choix du terrain et l'implantation des vignobles alignés avec des pratiques de viticulture durable. Par rapport au Brésil, le pays a une bonne tradition de 
Tableau 1. Principaux cadres de Viticulture Durable dans le contexte international.

\begin{tabular}{|c|c|c|}
\hline Pays & Cadre & Escope \\
\hline \multirow[t]{3}{*}{ Afrique du Sud } & Sustainable Wine South Africa (SWSA) & $\begin{array}{l}\text { Initiative qui intègre les programmes IPW et BWI } \\
\text { et les institutions responsables. Le label « Integity } \\
\text { \& Sustainability » identifie le respect aux critères } \\
\text { de IPW et il est un outil de traçabilité [11]. }\end{array}$ \\
\hline & Integrated Production of Wine (IPW) & $\begin{array}{l}\text { Établie en 1998, ce programme inclut la } \\
\text { traçabilité, la production intégrée et les pratiques } \\
\text { de viticulture durable pour les caves et les } \\
\text { vignobles }[12,13] \text {. Environ } 95 \% \text { du volume total } \\
\text { de production du pays est certifié IPW et } 75 \% \\
\text { des producteurs [14]. }\end{array}$ \\
\hline & Biodiversity \& Wine Initiative (BWI) & $\begin{array}{l}\text { Partenariat entre l'industrie et WWF pour la } \\
\text { conservation du Cape Floral Kingdom et ses } \\
\text { surfaces naturels [15]. }\end{array}$ \\
\hline \multirow[t]{2}{*}{ Nouvelle Zélande } & Sustainable Winegrowing New Zealand (SWNZ) & $\begin{array}{l}\text { Programme national, depuis } 2002 \text {, c'est un guide } \\
\text { que fait la proposition d'un modèle de meilleures } \\
\text { pratiques dans les vignobles et dans les caves [16]. }\end{array}$ \\
\hline & CarboNZero & $\begin{array}{l}\text { Premier standard de certification pour la neu- } \\
\text { tralisation des gazes à effet de serre sous } \\
\text { la norme ISO14065. Le cadre comprend un } \\
\text { outil pour identifier l'empreinte de charbon et } \\
\text { l'option d'achats des crédits pour neutraliser des } \\
\text { émissions [17]. }\end{array}$ \\
\hline Australie & $\begin{array}{l}\text { McLaren Vale Sustainable Winegrowing Australia } \\
\text { (MVSWGA) }\end{array}$ & $\begin{array}{l}\text { Programme lancé en } 2009 \text { qui est basé sur auto- } \\
\text { évaluation et sur la proposition d'amélioration } \\
\text { constante. Le programme compte avec environ } \\
35 \% \text { des producteurs et } 40 \% \text { de la surface de la } \\
\text { McLaren Vale [18]. }\end{array}$ \\
\hline Chili & $\begin{array}{l}\text { National Code of Sustainability for Chilean Wine } \\
\text { Industry }\end{array}$ & $\begin{array}{l}\text { Supporté par le consortium Vinos de Chile, depuis } \\
2011 \text {, le programme certifie en trois axes : le } \\
\text { vignoble, la cave et les pratiques de gestion [19]. }\end{array}$ \\
\hline $\begin{array}{l}\text { Etats-Unis } \\
\text { (Californie) }\end{array}$ & $\begin{array}{llll}\begin{array}{l}\text { California } \\
\text { (CSWA) }\end{array} & \text { Sustainable } & \text { Winegrowing } & \text { Alliance } \\
\text { (CSW } & & \end{array}$ & $\begin{array}{l}\text { Le programme comprend un guide détaillé } \\
\text { (California Code of Sustainable Winegrowing } \\
\text { Workbook), avec } 15 \text { chapitres, ainsi qu'un } \\
\text { programme d'entrainement et la certification } \\
\text { (Certified California Sustainable Winegrowing - } \\
\text { CCSW) [20]. }\end{array}$ \\
\hline France & Terra Vitis & $\begin{array}{l}\text { Reconnue par le Ministère de l'Agriculture de } \\
\text { la France, le programme utilise les directives } \\
\text { de l'agriculture raisonnée ; il est structuré dans } \\
\text { une fédération avec les associations locales } \\
\text { de : Bourgogne-Beaujolais, Loire, Bordeaux, } \\
\text { Champagne et Rhône-Méditerranée [21]. }\end{array}$ \\
\hline
\end{tabular}

discussion et des propositions méthodologiques par rapport au développement durable. Cela peut être illustré, par exemple, par la présence des discussions internationales (comme la Rio 92 et le Forum Social Mondial), la participation pour l'élaboration de la ISO26000 (norme pour Responsabilité Sociale) ou les importants indices de recyclage de l'aluminium et des emballages des produits agricoles chimiques, que le pays occupe la première position. Par contre, en ce qui concerne la viticulture durable, le Brésil a des initiatives isolées et non des propositions systématisées.

La deuxième phase a été dédiée à l'organisation des données. Les principales techniques étaient : l'analyse statistique descriptive, l'analyse des discours et la production de cartes thématiques et conceptuelles. La troisième phase comprend la proposition du cadre et sa validation avec l'application dans les deux régions viticoles recherchées. Plus d'information sur le méthode utilisée dans la recherche peut être trouvée dans les documents de la bibliographie [22].

\section{BaccuS}

Le BaccuS a été conçu pour être un outil flexible et adaptable à des contextes différents - pouvant représenter différents territoires, ou des acteurs avec différents degrés d'avancement dans les pratiques de viticulture durable. Le cadre est structuré sous une forme matricielle, mettant en rapport les cinq dimensions du développement durable et quatre lignes directives, qui sont articulées par thèmes, comme on le voit dans la figure ci-dessous (Fig. 1). Cette structure permet d'observer individuellement les enjeux, les relations et les impacts.

Les dimensions du développement durable - environnemental, économique, social, politique-institutionnel, territorial - peuvent être considérées comme des perspectives de lecture de la réalité, ou d'appropriation de la notion de durabilité dans un contexte donné. Les directives sont des orientations pour l'action. À l'origine, les directives sont des principes généraux qui peuvent être appliquées dans différents contextes ; cependant, la 


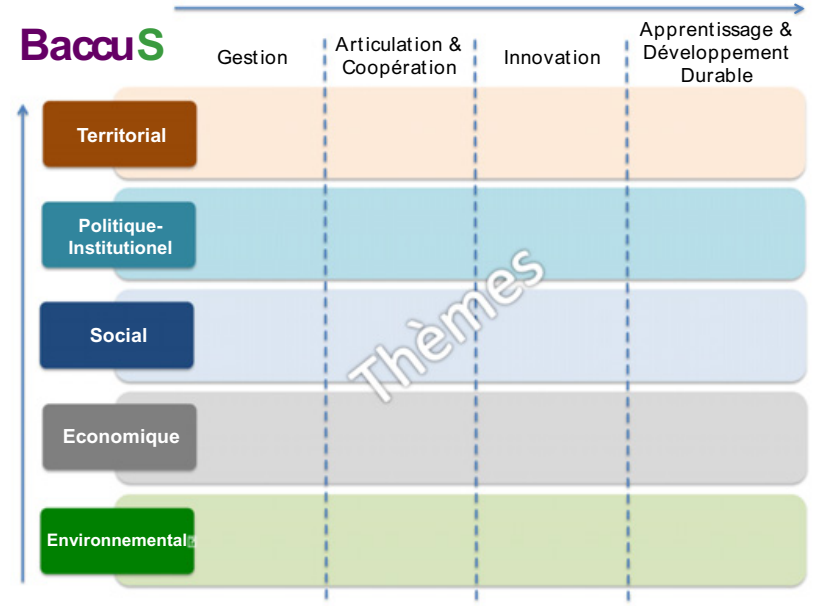

Figure 1. Schéma du cadre BaccuS. Source : Elaborée par S.S. Flores.

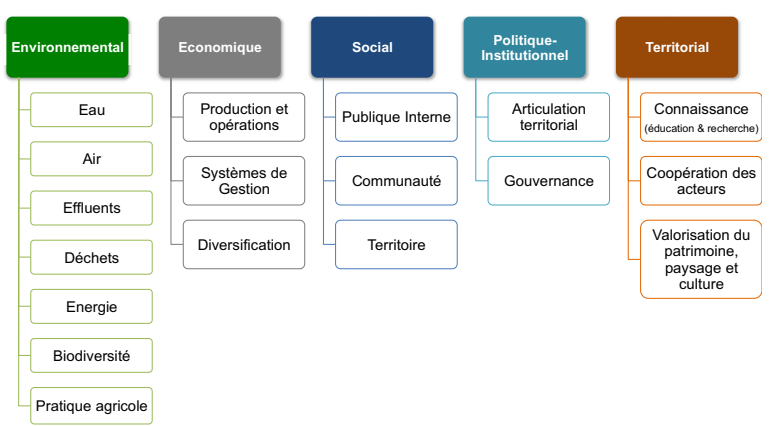

Figure 2. Les dimensions et les thèmes du BaccuS. Source : Elaborée par S.S. Flores.

recherche a montré que le parcours pour internaliser les principes de développement durable et de faire progresser le traitement du sujet, au sens large, passe par l'application de ces quatre lignes directrices - gestion, articulation et coopération, innovation, apprentissage et développement durable.

Pour articuler les dimensions et les directives, 18 thèmes sont proposés, organisés à partir des dimensions (Fig. 2). Les thèmes peuvent être compris comme une « vision pratique $»$ : ils sont autant d'enjeux dans lesquels les dimensions et les lignes directives peuvent être matérialisées et transformées en actions. Aussi, les thèmes mettent en rapport la théorie et la pratique, dans la mesure où ils transforment des orientations générales en initiatives concrètes et des indicateurs de contrôle, ou des facteurs qui doivent être considérés pour établir des initiatives envisageant le développement durable. Encore, les thèmes contextualisent les dimensions et les directives dans le(s) territoire(s) en question. Le format permet une vision d'ensemble et, en même temps, une lecture simplifiée en ordre croissant de complexité des dimensions et des lignes directrices, indiquée par les flèches dans le diagramme (Fig. 1), ce qui implique une fonction didactique suggérant un parcours pour renforcer la performance en matière de développement durable.

\subsection{Directives : processus pour le développement durable}

Souvent, les protocoles liés au développement durable utilisent des dimensions (ou des chapitres, axes) qui sont détaillées en indicateurs - aussi, les indicateurs peuvent être mieux spécifiés en paramètres. Dans le BaccuS, l'objectif est d'avancer et d'identifier : des facteurs qui serraient déterminants pour le succès dans l'intégration des principes du développement durable ; ou des éléments dont l'absence serait configurée comme une barrière pour y avancer. Dans ce sens, les directives ont été pensées comme des « conditionnes d'existence», ou un guide pour que les acteurs et les territoires se développent pleinement, à la recherche de la durabilité dans un sens large, compte tenu de la continuité et de l'équilibre. Les directives sont organisées dans un ordre croissant de complexité.

Le processus démarre avec la gestion, ce qui comprend l'organisation et les opérations, surtout à l'échelle des caves. La gestion implique dans la formalisation des processus, l'établissement des contrôles pour l'évaluation et le suivi, ainsi que la promotion de la planification, de la professionnalisation et de la communication avec les parties prenantes. Ces principes sont des bases pour une performance plus efficace vers des objectifs de développement durable envisagés.

Dans un deuxième niveau, la directive d'articulation et de coopération implique d'autres acteurs, soit des caves, soit des institutions de support, dans l'établissement des initiatives communes ou les simples échanges d'information. Elles reflètent la construction des liens et des relations qui sont à la base de la consolidation des territoires du vin. La directive peut faire référence à l'acteur lui-même, dans sa relation avec d'autres agents, ou au territoire, ce qui touche à la fois son organisation interne, ainsi que les relations avec les acteurs externes. Au niveau initial, il apparaît l'échange d'informations, en outre, la coopération peut évoluer vers des initiatives de formation et de qualification, de promotion des produits, du territoire ou de l'œnotourisme, dans le développement ou l'amélioration des produits et des processus, ainsi que l'optimisation de l'infrastructure de production. La coopération est institutionnalisée dans les Associations. Les réseaux formés contribuent à la dynamisation du territoire en favorisant la mise à jour des informations et en créant une synergie qui contribue à la résilience, ainsi que leurs perspectives de développement, en termes quantitatifs ou qualitatifs.

La directive de l'innovation favorise une réflexion sur les produits, les processus et l'activité des acteurs, sur les moyens possibles de faire les choses, ce qui incite un changement de paradigmes. La structuration des acteurs et des territoires, ce qui implique la gestion, ainsi que l'articulation et la coopération, sont les bases pour promouvoir l'innovation. Si l'innovation favorise le développement durable, ce dernier favorise les processus d'innovation, en niveau des acteurs ou des territoires, et elle est considérée comme une nouvelle frontière pour l'innovation. Il est important de considérer que l'innovation n'est pas seulement technique, mais peut être aussi organisationnel et/ou sociale.

Finalement, la directive de apprentissage et développement durable reflète l'idée que le développement durable est un concept et une approche en constante 
évolution, comme les territoires eux-mêmes. L'apprentissage et le développement durable sont considérés comme indissociables. L'apprentissage se fait à la fois individuellement et aussi dans la perspective du territoire. L'apprentissage peut se produire en termes techniques ou dans d'articulation des réseaux, ainsi que dans sa gestion [25]. Dans tous les cas, l'apprentissage devrait se traduire par un changement de comportement des acteurs, qui se reflète dans les produits, les processus, la gestion et les relations sur le territoire ou à l'extérieur. Il est une conséquence des directives précédentes et également une garantie que les progrès réalisés continueront en tant que un processus individuel ou collectif, qu'impact sur le territoire et sur les territorialités établis. Dans un sens plus large, la construction de la durabilité territoriale passe par un apprentissage collectif, y compris l'alignement des pratiques et la participation des parties prenantes.

\subsection{Dimensions et thèmes}

Dans le même sens que les directives, les dimensions sont aussi organisées par rapport à un ordre de complexité. Chaque dimension a des thèmes qui sont responsables de l'articulation avec les lignes directrices, en établissant un dialogue avec le territoire et ses enjeux. Les indicateurs ont été choisis en tenant compte à la fois des programmes et des protocoles de viticulture durable analysés, mais aussi des initiatives déjà en cours.

Le format matriciel adopté permet de visualiser les dimensions dans son ensemble, montrant les principales relations entre les indicateurs. Les relations sont exprimées sous la forme de flèches, reliant les indicateurs au sein de la même directive, ou vers les directrices plus complexes. L'option est justifiée par le fait que les lignes directrices indiquent un processus ou une manière dont les progrès sont assurés par le renforcement des directives précédentes.

L'analyse a identifié aussi des indicateurs de synthèse pour chaque dimension (soulignés en orange dans les diagrammes suivants). Les indicateurs de synthèse systématisent les enjeux et les thèmes pour chaque dimension ; en même temps ils ont le potentiel d'établir un dialogue avec d'autres dimensions. Les indicateurs de synthèse représentent, donc, des enjeux centraux, que peuvent motiver des programmes spécifiques et avoir un effet transversal, avec des répercutions dans d'autres thèmes. Dans ce moment, la construction du protocole était jusqu'au niveau des indicateurs, et les paramètres n'ont pas été définis. La perspective est que les paramètres sur chacun des thèmes et des indicateurs peuvent être développés à l'avenir, à partir de bases techniques et d'une plus grande participation des parties prenantes, de manière à être alignés avec des objectifs des territoires et des acteurs.

\subsubsection{La dimension environnementale}

Placer le point de vue de l'environnement dans la base se justifie par plusieurs raisons, parmi lesquelles figurent le besoin de prioriser les enjeux liés à la problématique de l'environnement et la possibilité de générer des indicateurs objectifs pour le suivi et l'évaluation. Sans surprise, la dimension environnementale est souvent citée

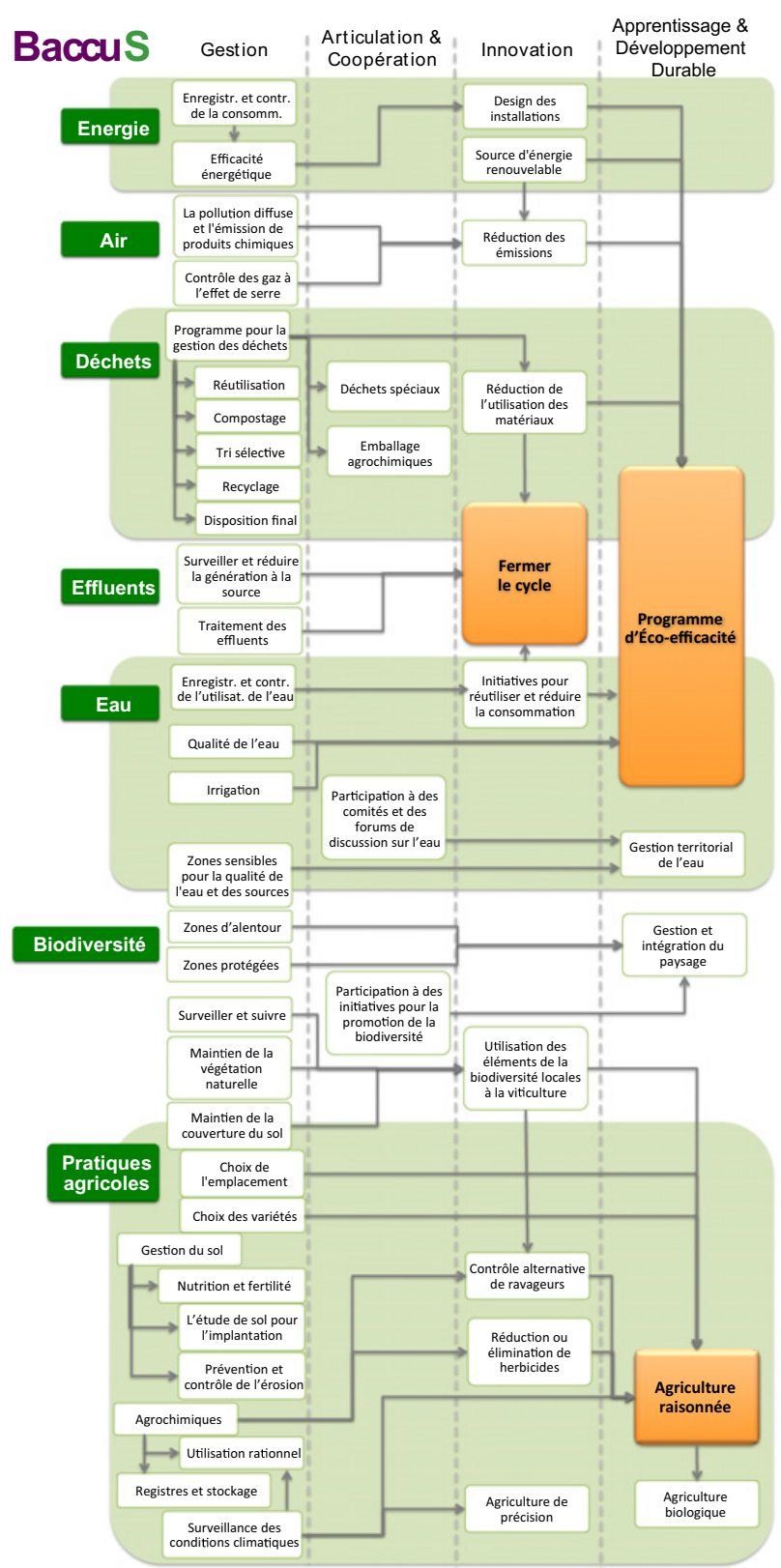

Figure 3. Thèmes et indicateurs dans la dimension environnementale. Source : Elaborée par S.S. Flores.

et joue un rôle prédominant dans les protocoles liés au développement durable.

À partir de l'approche territoriale utilisée, la dimension environnementale du développement durable est liée à la réduction de l'empreinte écologique, en réduisant la consommation des ressources naturelles (tels que l'eau et l'énergie), améliorant la qualité du produit (par rapport à l'environnement et la culture) et restaurant l'écosystème local. Les indicateurs pour cette dimension sont en général liés à l'utilisation des ressources naturelles et à l'impact environnemental (comme par exemple : l'eau, l'énergie, la biodiversité, les déchets et la pollution). Dans le contexte de la viticulture, d'autres possibilités sont les zones alentours, l'utilisation des engrais chimiques et la qualité du produit. Dans le diagramme suivant (Figure 3), il est possible d'observer les indicateurs proposés dans le BaccuS. 


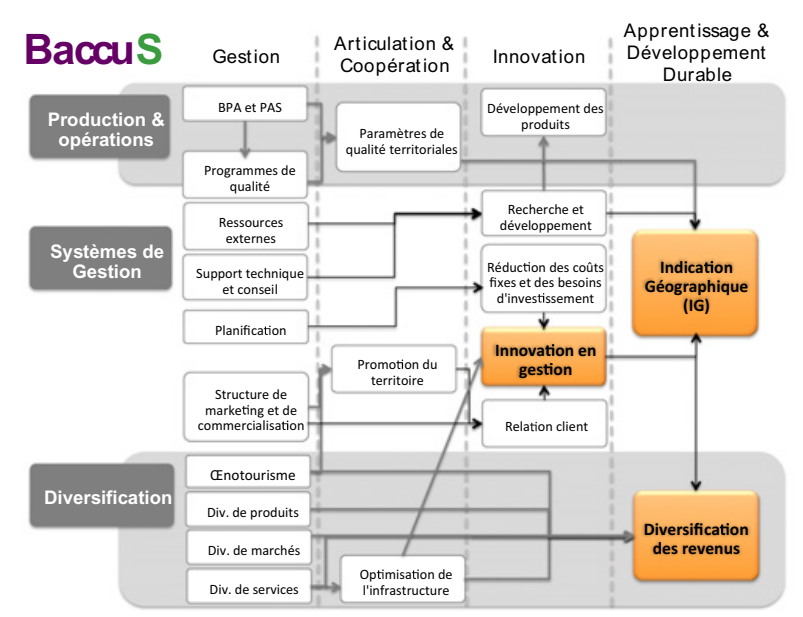

Figure 4. Thèmes et indicateurs dans la dimension économique. Source : Elaborée par S.S. Flores.

\subsubsection{La dimension économique}

Le facteur économique, comme la dimension environnementale, occupe une position importante dans les discours des acteurs, même si, en général, les protocoles liés au développement durable, sont fragiles dans la dimension économique, et se finissent par être parties des autres types de rapports. Dans le BaccuS, l'option était de mettre en évidence cette dimension étant donné son importance surtout si l'on veut bien considérer pour les territoires du vin émergents.

Concernant l'approche utilisée dans cette recherche, la dimension économique fait référence à la production de valeur ajoutée territoriale, en valorisant le territoire et l'identité qui alimentent le système économique local. En outre, la dimension fait référence à la capacité des producteurs en termes de potentialités locales, en conférant de la typicité aux produits et permettant la différentiation de la communication. Des indicateurs possibles dans cette dimension sont des produits de terroir, qui font référence à des caractéristiques locales, des labels et des marques locales, générant des emplois et de la diversification au sens large du terme. Dans le diagramme suivant (Fig. 4), il est possible d'observer les indicateurs proposés pour la dimension économique dans le BaccuS, organisés selon trois thèmes : production-opération, systèmes de gestion et diversification.

La production et les opérations $(\mathrm{P} \& \mathrm{O})$ viennent en premier, car le thème est considéré comme l'étage initial pour l'organisation des activités et des ressources, en favorisant une gestion de l'environnement plus efficace. Le thème des systèmes de gestion doit être vu en synergie avec celle de la production et les opérations; alors que la $\mathrm{P} \& \mathrm{O}$ focalise le processus productif, les systèmes de gestion se concentrent sur la planification et les enjeux qui ont une interface externes, concernant l'allocation des ressources et des décisions stratégiques allant de la promotion du territoire et des produits aux investissements et à la gestion des coûts. Dans ce sens, l'Indication Géographique (IG) est importante pour l'articulation et la planification vis-àvis du territoire.

\subsubsection{La dimension sociale}

La dimension sociale du développement durable représente à la fois la qualité de vie et les indicateurs sociaux qui passent par l'établissement des relations éthiques

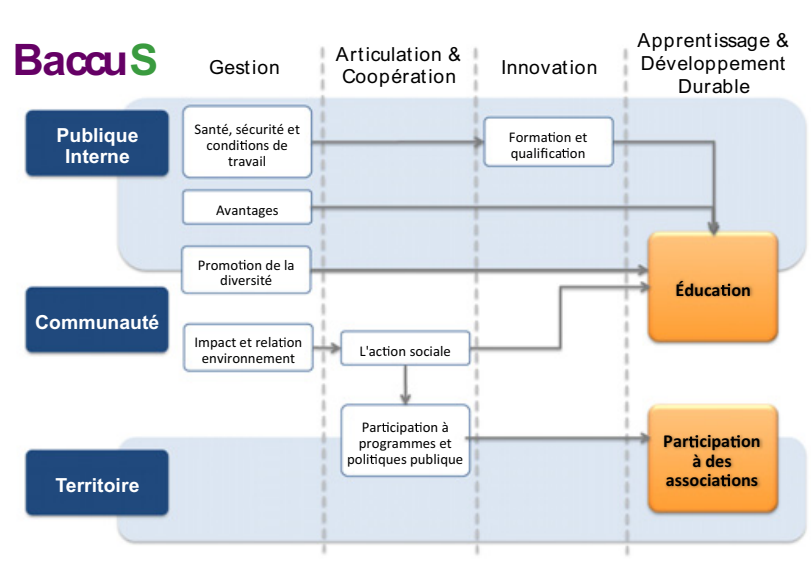

Figure 5. Thèmes et indicateurs dans la dimension sociale. Source : Elaborée par S.S. Flores.

avec les stakeholders, de la micro-échelle à la macroéchelle. Aussi, la dimension comprend la question de la participation dans les associations du territoire. Dans ce sens, en valorisant les enjeux sociaux eux-mêmes, la dimension a un rôle important de transition pour discuter des questions plus liées au territoire et ouvre la discussion par rapport à la prochaine dimension, la politique-institutionnelle.

Dans le diagramme (Fig. 5), il est possible d'observer les indicateurs proposés pour la dimension sociale dans le BaccuS. La dimension propose trois thèmes qui sont en réalité des échelles d'action. La première est le publique interne, qui comprend les conditions de travail et la promotion de la diversité. Dans un deuxième niveau, la communauté représente les relations avec l'environnement et aussi l'action sociale. L'éducation est un indicateur de synthèse qui considère les actions par rapport aux employés et aussi le support à des initiatives et à des institutions dans le territoire.

Dans le troisième niveau, le territoire souligne la participation et le support des programmes et politiques publiques, ainsi que l'implication dans les associations locales. Dans ce sens, la participation comprend les institutions qui représentent les territoires du vin, mais aussi des associations professionnels, des clubs et des services, entre autres.

\subsubsection{La dimension politique-institutionnelle}

La dimension politique-institutionnel met l'accent sur l'action des associations et des instances représentatives par rapport à des enjeux et des contextes externes. C'est la capacité des acteurs du territoire de s'organiser et de prendre des décisions, concernant des influences externes, sur des questions telles que la planification et l'appropriation des ressources. Aussi, la dimension comprend des enjeux liés à l'organisation institutionnelle du territoire par les réseaux des relations, mais aussi des syndicats, des coopératives et des associations. Les indicateurs proposés pour la dimension politique-institutionnelle dans le BaccuS peuvent être observés dans le diagramme suivant (Fig. 6).

Les deux thèmes sont la gouvernance et l'articulation territoriale. Dans le BaccuS, il est observé d'abord la gestion et l'organisation familiale, car le secteur compte 


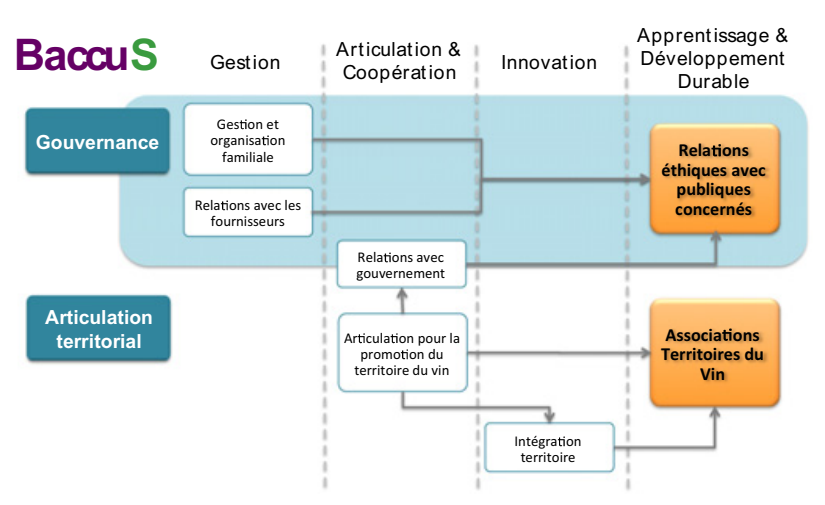

Figure 6. Thèmes et indicateurs dans la dimension politiqueinstitutionnelle. Source : Elaborée par S.S. Flores.

avec une participation importante des entreprises de base familiale. L'indicateur de synthèse des relations éthiques avec les publics concernés systématise le thème et peut être considéré comme un synonyme de l'approche de la responsabilité sociale. De plus, il y a une relation directe avec l'indicateur des relations avec le gouvernement, ce qui inclut la participation dans la gestion publique et les forums de discussions, le support à des politiques publiques, ainsi que des pratiques anti-corruption.

\subsubsection{La dimension territoriale}

La dimension territoriale a été choisie en dernière place dans le BaccuS en raison de ses caractéristiques. D'une part, la dimension peut être considérée la plus complexe dans l'approche et la systématisation des indicateurs, d'autre part la dimension territoriale a une nature transversale et d'intégration, qui nécessite notamment le développement des autres dimensions pour sa propre évolution. La dimension est liée à la capacité de promouvoir la reproduction et le développement de l'identité, en respectant des conditions locales. Dans ce cas, il y a un lien direct à la capacité de la viticulture de s'intégrer au local et à illustrer les liens entre les caractéristiques des produits et l'identité du territoire. Cette dimension systématise et crée des relations avec les dimensions antérieures. Ainsi, la dimension territoriale ne peut pas être travaillé de manière isolée, mais les thèmes ont la tendance à induire un regard transversal sur les autres dimensions, après tout, le territoire est à la base, mais aussi l'articulation.

Les indicateurs proposés pour la dimension territoriale sont dans le diagramme suivant (Fig. 7). L'articulation des thèmes dans le BaccuS donne la priorité à une réflexion sur les facteurs qui contribuent au développement des acteurs et à leur capacité de reproduction dans le territoire. Parmi les cinq indicateurs de synthèse, quatre reprennent d'autres dimensions, ce qui renforce le caractère transversal des thèmes travaillés.

Le premier thème met en évidence la (re)connaissance du territoire, dans le sens de la promotion des recherches, ainsi que des initiatives pour encourager la génération des connaissances sur le territoire. Encore, le thème comprend la systématisation des informations et sa disponibilité au niveau des acteurs, mais aussi au niveau du territoire. Il est souligné le rôle de l'éducation $\mathrm{du}$ territoire pour son développement. Parallèlement, la coopération entre les acteurs apparaît dans les sens des échanges des informations, qui peuvent évoluer par des

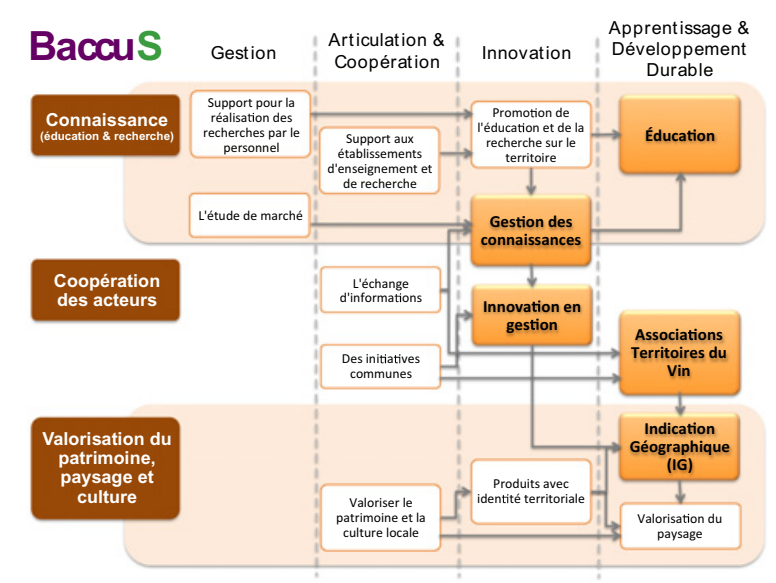

Figure 7. Thèmes et indicateurs dans la dimension territoriale. Source : Elaborée par S.S. Flores.

initiatives communes. Ces démarches vont demander des compétences de gestion supérieures, ce qui doit impliquer dans l'innovation en gestion.

Finalement, la valorisation du patrimoine, du paysage et de la culture peut apparaître dans des initiatives individuelles, à partir de la promotion des produits qui représentent l'identité territoriale. Par contre, son application effective passe pour l'articulation des acteurs et les initiatives collectives, envisageant la valorisation des ressources territoriales et du patrimoine dans un sens large - en considérant l'environnement, mais aussi, les personnes, la culture et l'identité. Une synergie qui est synonyme du développement durable, vu à partir du territoire.

\section{Considérations}

Le développement durable est en enjeu important qui exige, en même temps, l'avancement avec nos propositions théoriques et méthodologiques, ainsi que dans les outils et indicateurs d'évaluation et contrôle. C'est un défi et une condition pour la maintenance des territoires. La motivation principale pour le développement du cadre BaccuS était de fournir un outil pour promouvoir l'amélioration de la performance des vignerons et des caves vis-à-vis les principes du développement durable, en aidant aux acteurs dans la prise de décision et à éclairer le concept dans les activités quotidiennes.

Le cadre BaccuS a deux contributions principales en considérant les autres protocoles étudiés : la structuration en lignes directrices et la perspective territoriale. Tandis que la plupart des protocoles sont structurés en dimensions (ou chapitres), qui sont détailles en indicateurs et paramètres, le BaccuS articule les dimensions avec les directives, qui proposent un processus d'apprentissage et de développement dans les producteurs et/ou dans les territoires. Ainsi, chaque indicateur est travaillé dans une échelle de complexité, dans l'indicateur lui-même, mais aussi en considérant les directives. Encore, la structure adoptée peut être utilisée pour des acteurs de grande ou de petite structure et de niveaux différents de formalisation dans les processus de gestion ; il contribue non seulement à une auto-évaluation, mais avec l'action de la vue des perspectives c'action afin d'assurer sa pérennité.

La perspective territoriale a guidé le développement de la recherche et elle est présente dans BaccuS en termes d'échelle, de champ et de structure. Considérant l'échelle, 
le protocole peut être utilisé pour évaluer le niveau du producteur ou du territoire, permettant une vision multiéchelle, mais aussi d'examiner les synergies dans les initiatives proposées. D'autre part, plusieurs indicateurs dans toutes les dimensions du développement durable guident la relation entre l'acteur et le territoire, allant de la participation à des projets locaux, à des Associations, à la coopération entre les acteurs pour promouvoir le territoire du vin. Dans le même temps, l'articulation et la coopération sont considérées comme des conditions pour évoluer dans les thèmes, ce qui intègre la structure $\mathrm{du}$ protocole. Cet approche apporte une contribution au développement durable du territoire du vin, à partir de sa territorialisation et reproduction de l'identité, qui ne se limite pas à la dimension territoriale, mais qui intègre les dimensions environnementales, économiques, sociales et politiques-institutionnelles.

Le cadre présente des limites en ce qui concerne sa portée et sa profondeur. Même avec un référentiel empirique pertinent, la recherche a examiné seulement deux territoires du vin au Brésil, donc, les conclusions ont une portée limitée puisqu'il manque les régions viticoles brésiliennes comme la Serra Gaúcha, ou le Planalto Catarinense. Par rapport à la profondeur, le BaccuS a avancé dans la proposition de la structure et des thèmes, mais il n'a pas détaillé les indicateurs dans des échelles ou des indices qui permettaient une évaluation quantitative, par exemple. Dans ce cas, il est considéré que le développement des indicateurs nécessite la participation d'une équipe multidisciplinaire, qui fournit une expertise technique pour aider les décisions prises. En plus, l'avance $\mathrm{du}$ protocole et de son application effective passe par l'implication des acteurs concernés.

Plus qu'une réponse fermée, pensez viticulture durable peut être la recherche d'un idéal plus lointain, ou aussi, un repère à l'horizon qui indique la voie à la durabilité du territoire. Une réflexion qui doit nécessairement être accompagnée par des initiatives d'action, par l'amélioration des pratiques existantes et par la proposition des paramètres et des pratiques alternatives, afin de consolider les territoires de vin et d'assurer son développement durable dans un sens large - en considérant ses dimensions environnementale, économique, sociale, politique-institutionnelle et territoriale.

\section{Références}

[1] R. Carson, Silent spring (Mariner Book, 1962)

[2] I. Sachs, Environnement et styles de développement. Ann. Économies, Sociétés, Civilisations. 29 e année 3, 553-570 (1974)

[3] United Nations. Our Common Future: Report of the World Commission on Environment and Development. (1987) at <http://conspect.nl/ pdf/Our_Common_Future-Brundtland_Report_ $1987 \cdot \mathrm{pdf}>$

[4] E. Leff, Epistemologia ambiental. (Cortez, 2010)

[5] E. Leff, Racionalidade ambiental (Civilização Brasileira, 2006)

[6] J. Theys, in Développement durable Territ. (ZUINDEAU, B.) 9-12 (Presse Universitaires Septentrion, 2010)
[7] A. Magnaghi, The urban village?: a charter for democracy and local self-sustainable development (Zed Books, 2005)

[8] Ministeère de l'Agriculture e la Peche et de la Ruralité. Guide pour une protection durable de la vigne: stratégie de protection pour une utilisation raisonnée et durable des intrants phytosanitaires en viticulture (2005)

[9] FAO. World Summit on Sustainable Development - Welcome. (2013) at <http://www.fao.org/ wssd/sard/index-en.htm>

[10] OIV. Resolution CST 1/2008 - OIV guidelines for sustainable vitiviniculture: production, processing and packaging of products (2008)

[11] SWSA. SWSA: sustainable wine South Africa. (2013) at <http://www.swsa.co.za>

[12] IPW. IPW certification policy. 4 (2008) at $<$ http://www.ipw.co.za/index.php>

[13] IPW. IPW manual for biodiversity. 14 (2006) at <http://www.ipw.co.za/index.php>

[14] IPW. The integrated production of wine scheme (IPW). (2013) at <http://www . ipw. co.za>

[15] WWF. Biodiversity and wine initiative: WWF South Africa (2013) at <www.bwi.co.za/?>

[16] SWNZ. Sustainable Winegrowing New Zealand Standards. (2013) at <nzwine.com/sustainability>

[17] NZWC. Summary of carboNZero certification?: the New Zealand Wine Company Limited. (2013) at $<$ http://www.carbonzero.co.nz/documents/ disclosure_nzwc_0809.pdf $>$

[18] MVSWGA. McLaren Vale Sustainable winegrowing Australia system (MVSWGA). (2013) at $<$ http://www. sustainablewinegrowing. com.au $>$

[19] Vinos de Chile. Código nacional de sustentabilidad de la industria vitivinícola chilena. (2014) at $<$ http://www.sustentavid.org >

[20] CSWA. California Code of Sustainable Winegrowing Workbook. (California Sustainable Winegrowing Alliance (CSWA), 2012) at <www.sustaina blewinegrowing.org/swpworkbook.php >

[21] TVBB. Cahier des charges production viticole durable et oenologie: Terra Vitis Beaujolais Borgogne (2012)

[22] S. S. Flores, Vitivinicultura sustentável no contexto do Brasil: uma proposta de abordagem (2015)

[23] I. Pierot, J. Rochard, Développement durable viticole: état de lieux. IFV Lett. Electron. oct 2013 1-8 (2013) at < http://www.vignevin.com/ fileadmin/users/ifv/actualites/lettre avril_2013/Lettre_Juillet13/2013-057_ Etat_des_lieux_du_durable.pdf $>$

[24] M. A. Pratt, Comparison of sustainability programs in the wine industry. in Proc. Int. Conf. Innov. Trends Wine Manag (Burgundy School of Business, 2012)

[25] M. Barbier, B. Elzen, System innovations knowledge regimes and design practices toward transitions for sustainable agriculture (2012) at <http:// www4.inra.fr/sad_eng/Publications2/Freee-books/System-Innovations-for-Sustain able-Agriculture> 